\title{
Hybrid classical-quantum fitting attention states to statistical mechanics of neocortical interactions
}

\author{
Lester Ingber ${ }^{1}$
}

\begin{abstract}
:
Hybrid Classical-Quantum computing has already arrived at several commercial quantum computers, offered to researchers and businesses. Here, application is made to a classical-quantum model of human neocortex, Statistical Mechanics of Neocortical Interactions (SMNI), which has had its applications published in many papers since 1981. However, this project only uses Classical (super-)computers.

Since 2015, a path-integral algorithm, PATHINT, used previously to accurately describe several systems in several disciplines, has been generalized from 1 dimension to $\mathrm{N}$ dimensions, and from classical to quantum systems, qPATHINT. Published papers have described the use of qPATHINT to neocortical interactions and financial options.

The classical space described by SMNI applies nonlinear nonequilibrium multivariate statistical mechanics to synaptic neuronal interactions, while the quantum space described by qPATHINT applies synaptic contributions from $\mathrm{Ca} 2+$ waves generated by astrocytes at tripartite neuron-astrocyte-neuron sites.

Previous SMNI publications since 2013 have calculated the astrocyte Ca2+ wave synaptic interactions from a closed-form (analytic) expression derived by the Principal Investigator. However, more realistic random shocks to the Ca2+ waves from ions entering and leaving these wave packets should be included using qPATHINT between electroencephalographic (EEG) measurements which decohere the quantum wave packets.

This current project extends calculations to multiple scales of interaction between classical events and expectations over the $\mathrm{Ca} 2+$ quantum processes to include these random shocks in previous codes used to fit EEG data to the SMNI model, that included the analytic forms for the quantum processes but now replaced by qPATHINT. The Principal Investigator's Adaptive Simulated Annealing (ASA) importance-sampling optimization code is used for fitting the combined classical-quantum system. Gaussian Quadratures is used for numerical calculation of momenta expectations of the astrocyte processes that contribute to SMNI synaptic interactions.

This project thereby demonstrates how some hybrid classical-quantum systems may be calculated quite well using only classical (super-)computers.
\end{abstract}

Key words: path integral, quantum systems, neocortical interactions

\section{Introduction}

\section{$1.1 \quad$ Hybrid computing}

There are several companies now offering commercial-grade Hybrid Classical-Quantum computers that can be accessed via the Cloud, e.g., Rigetti, D-Wave, Microsoft, and IBM (Ingber, 2021a); see

https://docs.ocean.dwavesys.com/projects/hybrid/en/latest/index.html

https://www.rigetti.com/what

\footnotetext{
${ }^{1}$ Corresponding author: Lester Ingber

email: ingber@caa.caltech.edu

Additional information may be obtained at https://www.ingber.com.
} 
https://azure.microsoft.com/en-us/solutions/hybrid-cloud-app/\#overview https://www.ibm.com/it-infrastructure/z/capabilities/hybrid-cloud

These Hybrid computers typically offer Classical computers to run optimization program on parameters in systems that are described by quantum variables using Quantum computers (Benedetti et al., 2019). Software for quantum states also has been developed, e.g., Tensorflow, the popular endto-end open-source tool for machine learning, also has been adapted for Hybrid classical-quantum computing, as described in

https://quantumzeitgeist.com/tensorflow-for-quantum-hits-first-birthday/

https://www.tensorflow.org/quantum

The PI has accounts on D-Wave and Rigetti computers, but has not yet ported SMNI code to these Quantum computers for Hybrid computations.

This project essentially merges codes generated for two previous XSEDE grant proposals, "Electroencephalographic field influence on calcium momentum waves" and "Quantum path-integral qPATHTREE and qPATHINT algorithms". These codes both run on Classical computers and thereby define a Hybrid Classical-Quantum system that can be calculated solely on a Classical computer.

Previous papers have fit electroencephalographic (EEG) data using a model of quantum wavepackets composed of a specific class of $\mathrm{Ca}^{2+}$ ions that are (re-)generated at tripartite neuronastrocyte-neuron sites, thereby influencing synaptic interactions (Ingber, 2018). Since 2011 (Ingber, 2011, 2012a), both classical and quantum calculations have been shown to be consistent with an interaction between the momenta $\mathbf{p}$ of these wave-packets with a magnetic vector potential $\mathbf{A}$ that is generated by highly synchronous firings of many thousands of neocortical neurons during tasks requiring short-term memory (STM). A closed-form ("analytic") path-integral calculation of this quantum process developed a wave-function and an expectation value of $\mathbf{p}$ in the presence of $\mathbf{A}$, thereby defining an example of quantum interactions coupled to a macroscopic system.

An important reason for further addressing this particular system using these codes is to enable more realistic inclusion of shocks to the wave-packet wave-function due to the regenerative process of the wave-packet, e.g., due to collisions between $\mathrm{Ca}^{2+}$ ions in the wave-packet causing some ions to leave the wave-packet during its hundreds of msec lifetime, or as new ions enter from the astrocyte processes. These may be considered as random processes (Ross, 2012). The codes PATHTREE/qPATHTREE and PATHINT/qPATHINT have been designed to include shocks in the evolution of a short-time probability distribution over thousands of foldings (Ingber, 2016a, $2017 \mathrm{a}$ b).

\subsection{SMNI}

A model of neocortex, Statistical Mechanics of Neocortical Interactions (SMNI), was developed circa 1979 (Ingber, 1982, 1983) and regularly enhanced since by the process of fitting experimental data from short-term memory (STM) and electroencephalography (EEG), with more recent papers including such fits to attention data (Ingber, 2018) and affective data (Alakus et al., 2020; Ingber, 2021b).

\subsection{PATHINT}

As defined by the path-integral, a one-dimensional code developed to numerically propagate shorttime conditional probability distributions (Ingber et al. 1991; Wehner \& Wolfer, 1983a.b), was generalized to PATHINT, a code in N dimensions and applied in several disciplines (Ingber, 2000a; 
Ingber \& Nunez, 1995; Ingber et al., 1996; Ingber \& Wilson, 2000), and to PATHTREE (Ingber et al., 2001).

\section{4 qPATHINT}

The PATHTREE and PATHINT codes were generalized to quantum systems, yielding qPATHTREE and qPATHINT (Ingber, 2016a, 2017a,c).

\subsection{Organization of paper}

Section 2 further describes SMNI in the context of this project.

Section 3 further describes Adaptive Simulated Annealing (ASA) in the context of this project.

Section 4 further describes qPATHINT in the context of this project.

Section 5 describes how the calculation proceeds between SMNI and qPATHINT.

Section 6 describes performance and scaling issues.

Section 7 is the Conclusion.

\subsection{Caveat}

As stated previously in these projects (Ingber, 2018),

"The theory and codes for ASA and [q]PATHINT have been well tested across many disciplines by multiple users. This particular project most certainly is speculative, but it is testable. As reported here, fitting such models to EEG tests some aspects of this project. This is a somewhat indirect path, but not novel to many physics paradigms that are tested by experiment or computation."

\section{SMNI}

The project Statistical Mechanics of Neocortical Interactions (SMNI) has been developed in over $30+$ papers since 1981, scaling aggregate synaptic interactions to describe neuronal firings, then scaling minicolumnar-macrocolumnar columns of neurons to mesocolumnar dynamics, and then scaling columns of neuronal firings to regional (sensory) macroscopic sites identified in EEG studies (Ingber, 1982, 1983, 1984, 1985a, 1994).

The measure of the success of SMNI has been to discover agreement/fits with experimental data from various modeled aspects of neocortical interactions, e.g., properties of short-term memory (STM) (Ingber, 2012a), including its capacity (auditory $7 \pm 2$ and visual $4 \pm 2$ ), duration, stability, primacy versus recency rule (Ingber, 1984, 1985a, 1994, 1995, 2000b, 2012a; Ingber \& Nunez, 1995), EEG dispersion relations (Ingber, 1985b), as well other phenomenon, e.g., Hick's law (Hick, 1952; Ingber, 1999; Jensen, 1987), nearest-neighbor minicolumnar interactions within macrocolumns calculating rotation of images, etc (Ingber, 1982, 1983, 1984, 1985a, 1994). SMNI was also scaled to include mesocolumns across neocortical regions to fit EEG data (Ingber, 1997, 2012a.b, 2018; Ingber \& Nunez, 2010).

\subsection{XSEDE EEG Project}

The Extreme Science and Engineering Discovery Environment (XSEDE.org) project since February 2013, "Electroencephalographic field influence on calcium momentum waves," fit the SMNI model to EEG data, wherein ionic $\mathrm{Ca}^{2+}$ momentum-wave effects among neuron-astrocyte-neuron 
tripartite synapses modified parameterization of background SMNI parameters. Direct calculations in classical and quantum physics supported the concept that the vector magnetic potential of EEG from highly synchronous firings, e.g., as measured during selective attention, can directly interact with these momentum-waves, thereby creating feedback between these ionic/quantum and macroscopic scales (Ingber, 2012a,b, 2015, 2016b, 2017a,c, 2018; Ingber et al., 2014; Nunez et al. 2013).

\subsection{1 qPATHINT For SMNI}

The present qPATHINT code determines how the quantum regenerative process that defines $\mathrm{Ca}^{2+}$ wave-packets also may produce reasonable shocks to the waves without seriously damaging its coherence properties. A proof of principal of its use has been published (Ingber, 2017a).

\subsection{SMNI With A}

Previous papers have modeled minicolumns as wires which support neuronal firings, mainly from large neocortical excitatory pyramidal cells in layer V (of I-VI), giving rise to currents which in turn gives rise to electric potentials measured as scalp EEG (Ingber, 2011, 2012a; Nunez et al., 2013). This gives rise to a magnetic vector potential

$$
\mathbf{A}=\frac{\mu}{4 \pi} \mathbf{I} \log \left(\frac{r}{r_{0}}\right)
$$

which has a log-insensitive dependence on distance. In the brain, $\mu \approx \mu_{0}$, where $\mu_{0}$ is the magnetic permeability in vacuum $=4 \pi 10^{-7} \mathrm{H} / \mathrm{m}$ (Henry/meter), where Henry has units of $\mathrm{kg}-\mathrm{m}-\mathrm{C}^{-2}$, the conversion factor from electrical to mechanical variables. For oscillatory waves, the magnetic field $\mathbf{B}=\nabla \times \mathbf{A}$ and the electric field $\mathbf{E}=(i c / \omega) \nabla \times \nabla \times \mathbf{A}$ do not have this log dependence on distance. Thus, A fields can contribute collectively over large regions of neocortex (Ingber, 2012a,b, 2015, 2016b, 2017a,c, 2018; Ingber et al., 2014; Nunez et al., 2013). The magnitude of the current is taken from experimental data on dipole moments $\mathbf{Q}=|\mathbf{I}| \hat{\mathbf{z}}$ where $\hat{\mathbf{z}}$ is the direction of the current $\mathbf{I}$ with the dipole spread over $z$. $\mathbf{Q}$ ranges from $1 \mathrm{pA}-\mathrm{m}=10^{-12} \mathrm{~A}-\mathrm{m}$ for a pyramidal neuron (Murakami \& Okada, 2006), to $10^{-9}$ A-m for larger neocortical mass (Nunez \& Srinivasan, 2006). These currents give rise to $q \mathbf{A} \approx 10^{-28} \mathrm{~kg}-\mathrm{m} / \mathrm{s}$. The velocity of a $\mathrm{Ca}^{2+}$ wave can be $\approx 20-50 \mu \mathrm{m} / \mathrm{s}$. In neocortex, a typical $\mathrm{Ca}^{2+}$ wave of 1000 ions, with total mass $m=6.655 \times 10^{-23} \mathrm{~kg}$ times a speed of $\approx 20-50 \mu \mathrm{m} / \mathrm{s}$, gives $\mathbf{p} \approx 10^{-27} \mathrm{~kg}-\mathrm{m} / \mathrm{s}$. This yields $\mathbf{p}$ to be on the same order as $q \mathbf{A}$.

\subsubsection{Results Including Quantum Scales}

Without random shocks, the wave-function $\psi_{\mathrm{e}}$ describing the interaction of $\mathbf{A}$ with $\mathbf{p}$ of $\mathrm{Ca}^{2+}$ wave-packets was derived in closed form from the Feynman representation of the path integral using path-integral techniques (Schulten, 1999), modified here to include A.

$$
\begin{gathered}
\psi_{\mathrm{e}}(t)=\int d \mathbf{r}_{0} \psi_{0} \psi_{F}=\left[\frac{1-i \hbar t /\left(m \Delta \mathbf{r}^{2}\right)}{1+i \hbar t /\left(m \Delta \mathbf{r}^{2}\right)}\right]^{1 / 4}\left[\pi \Delta \mathbf{r}^{2}\left\{1+\left[\hbar t /\left(m \Delta \mathbf{r}^{2}\right)\right]^{2}\right\}\right]^{-1 / 4} \\
\times \exp \left[-\frac{\left[\mathbf{r}-\left(\mathbf{p}_{0}+q \mathbf{A}\right) t / m\right]^{2}}{2 \Delta \mathbf{r}^{2}} \frac{1-i \hbar t /\left(m \Delta \mathbf{r}^{2}\right)}{1+\left[\hbar t /\left(m \Delta \mathbf{r}^{2}\right)\right]^{2}}+i \frac{\mathbf{p}_{0} \cdot \mathbf{r}}{\hbar}-i \frac{\left(\mathbf{p}_{0}+q \mathbf{A}\right)^{2} t}{2 \hbar m}\right] \\
\psi_{F}(t)=\int \frac{d \mathbf{p}}{2 \pi \hbar} \exp \left[\frac{i}{\hbar}\left(\mathbf{p}\left(\mathbf{r}-\mathbf{r}_{0}\right)-\frac{\mathbf{\Pi}^{2} t}{(2 m)}\right)\right]=\left[\frac{m}{2 \pi i \hbar t}\right]^{1 / 2} \exp \left[\frac{i m\left(\mathbf{r}-\mathbf{r}_{0}-q \mathbf{A} t / m\right)^{2}}{2 \hbar t}-\frac{i(q \mathbf{A})^{2} t}{2 m \hbar}\right]
\end{gathered}
$$




$$
\psi_{0}=\psi\left(\mathbf{r}_{0}, t=0\right)=\left(\frac{1}{\pi \Delta \mathbf{r}^{2}}\right)^{1 / 4} \exp \left(-\frac{\mathbf{r}_{0}^{2}}{2 \Delta \mathbf{r}^{2}}+i \frac{\mathbf{p}_{0} \cdot \mathbf{r}_{0}}{\hbar}\right)
$$

where $\psi_{0}$ is the initial Gaussian wave-packet, $\psi_{F}$ is the free-wave evolution operator, $\hbar$ is the Planck constant, $q$ is the electronic charge of $\mathrm{Ca}^{2+}$ ions, $m$ is the mass of a wave-packet of 1000 $\mathrm{Ca}^{2+}$ ions, $\Delta \mathbf{r}^{2}$ is the spatial variance of the wave-packet, the initial momentum is $\mathbf{p}_{0}$, and the evolving canonical momentum is $\boldsymbol{\Pi}=\mathbf{p}+q \mathbf{A}$. Detailed calculations show that $\mathbf{p}$ of the $\mathrm{Ca}^{2+}$ wave-packet and $q \mathbf{A}$ of the EEG field make about equal contributions to $\boldsymbol{\Pi}$.

\subsection{2 $\mathrm{SMNI}+\mathrm{Ca}^{2+}$ wave-packet}

Tripartite influence on synaptic $B_{G^{\prime}}^{G}$ is measured by the ratio of the wave-packet's $<\mathbf{p}(t)>_{\psi * \psi}$ to $<\mathbf{p}_{0}\left(t_{0}\right)>_{\psi * \psi}$ at the onset of each attention task, measured just after each EEG measurement. Here $<>_{\psi * \psi}$ is taken over $\psi_{\mathrm{e}}^{*} \psi_{\mathrm{e}}$.

$$
<\mathbf{p}>_{\psi * \psi}=m \frac{<\mathbf{r}>_{\psi * \psi}}{t-t_{0}}=\frac{q \mathbf{A}+\mathbf{p}_{0}}{m^{1 / 2}|\Delta \mathbf{r}|}\left(\frac{(\hbar t)^{2}+\left(m \Delta \mathbf{r}^{2}\right)^{2}}{\hbar t+m \Delta \mathbf{r}^{2}}\right)^{1 / 2}
$$

A changes slower than $\mathbf{p}$, so static approximation of $\mathbf{A}$ used to derive $\psi_{\mathrm{e}}$ and $\langle\mathbf{p}\rangle_{\psi * \psi}$ was deemed reasonable to use within P300 EEG epochs, resetting $t=0$ at the onset of each classical EEG measurement (1.953 ms apart), using the current-time A. This permits tests of interactions across scales in a classical context.

This result contains $\hbar t$, thereby explicitly containing quantum $\hbar$ as well as explicit timedependence $t$, and is therefore directly testable. Note that this analytic (closed-form) solution is replaced in this project by qPATHINT, permitting shocks to astrocyte-(re-)generated $\mathrm{Ca}^{2+}$ wavepackets to be included in the fits to EEG data.

\subsubsection{SMNI Context}

A short description of the SMNI structure is required to understand how the qPATHINT development is used in this multiple-scale model (Ingber, 2016b).

After a statistical-mechanical aggregation of synaptic, neuronal and columnar scales (Ingber, 1982, 1983), the SMNI Lagrangian $L$ in the prepoint (Ito) representation is

$$
\begin{gathered}
L=\sum_{G, G^{\prime}}(2 N)^{-1}\left(\dot{M}^{G}-g^{G}\right) g_{G G^{\prime}}\left(\dot{M}^{G^{\prime}}-g^{G^{\prime}}\right) /(2 N \tau)-V^{\prime} \\
g^{G}=-\tau^{-1}\left(M^{G}+N^{G} \tanh F^{G}\right), g^{G G^{\prime}}=\left(g_{G G^{\prime}}\right)^{-1}=\delta_{G}^{G^{\prime}} \tau^{-1} N^{G} \operatorname{sech}^{2} F^{G}, g=\operatorname{det}\left(g_{G G^{\prime}}\right)
\end{gathered}
$$

where $G=\{E, I\}$ for chemically independent excitatory and inhibitory synaptic interactions, $N^{G}$ $=\left\{N^{E}=160, N^{I}=60\right\}$ was chosen for visual neocortex, $\left\{N^{E}=80, N^{I}=30\right\}$ was chosen for all other neocortical regions, $M^{G^{\prime}}$ and $N^{G^{\prime}}$ in $F^{G}$ are afferent macrocolumnar firings scaled to efferent minicolumnar firings by $N / N * \approx 10^{-3}$, and $N *$ is the number of neurons in a macrocolumn, about $10^{5} . \tau$ is usually considered to be on the order of 5-10 ms. $V^{\prime}$ includes nearest-neighbor mesocolumnar interactions (Ingber, 1982, 1983). The threshold factor $F^{G}$ is derived as

$$
F^{G}=\sum_{G^{\prime}} \frac{\nu^{G}+\nu^{\ddagger E^{\prime}}}{\left((\pi / 2)\left[\left(v_{G^{\prime}}^{G}\right)^{2}+\left(\phi_{G^{\prime}}^{G}\right)^{2}\right]\left(\delta^{G}+\delta^{\ddagger E^{\prime}}\right)\right)^{1 / 2}}
$$




$$
\begin{gathered}
\nu^{G}=V^{G}-a_{G^{\prime}}^{G} v_{G^{\prime}}^{G} N^{G^{\prime}}-\frac{1}{2} A_{G^{\prime}}^{G} v_{G^{\prime}}^{G} M^{G^{\prime}} \\
\nu^{\ddagger E^{\prime}}=-a_{E^{\prime}}^{\ddagger E} v_{E^{\prime}}^{E} N^{\ddagger E^{\prime}}-\frac{1}{2} A_{E^{\prime}}^{\ddagger E} v_{E^{\prime}}^{E} M^{\ddagger E^{\prime}} \\
\delta^{G}=a_{G^{\prime}}^{G} N^{G^{\prime}}+\frac{1}{2} A_{G^{\prime}}^{G} M^{G^{\prime}} \\
\delta^{\ddagger E^{\prime}}=a_{E^{\prime}}^{\ddagger E} N^{\ddagger E^{\prime}}+\frac{1}{2} A_{E^{\prime}}^{\ddagger E} M^{\ddagger E^{\prime}} \\
a_{G^{\prime}}^{G}=\frac{1}{2} A_{G^{\prime}}^{G}+B_{G^{\prime}}^{G}, a_{E^{\prime}}^{\ddagger E}=\frac{1}{2} A_{E^{\prime}}^{\ddagger E}+B_{E^{\prime}}^{\ddagger E}
\end{gathered}
$$

where $A_{G^{\prime}}^{G}$ is the columnar-averaged direct synaptic efficacy, $B_{G^{\prime}}^{G}$ is the columnar-averaged backgroundnoise contribution to synaptic efficacy. $A_{G^{\prime}}^{G}$ and $B_{G^{\prime}}^{G}$ have been scaled by $N * / N \approx 10^{3}$ keeping $F^{G}$ invariant. Other values are consistent with experimental data, e.g., $V^{G}=10 \mathrm{mV}, v_{G^{\prime}}^{G}=0.1 \mathrm{mV}$, $\phi_{G^{\prime}}^{G}=0.03^{1 / 2} \mathrm{mV}$. The “‡” parameters arise from regional interactions across many macrocolumns.

A Dynamic Centering Mechanism (DCM) model is used (Ingber, 2016b), wherein the $B_{G^{\prime}}^{G}$ are reset every few epochs of $\tau$, parameterized to include contributions from tripartite neuronastrocyte-neuron contributions. The DCM is consistent with experimental observations of shifts in synaptic activity during attention tasks (Briggs et al., 2013; Mountcastle et al. 1981 ). PATHINT also has been successfully used with the SMNI Lagrangian $L$ to calculate properties of STM for both auditory and visual memory (Ericsson \& Chase, 1982; Zhang \& Simon, 1985) calculating the stability and duration of STM, the observed $7 \pm 2$ capacity rule of auditory memory and the observed $4 \pm 2$ capacity rule of visual memory (Ingber, 2000a; Ingber \& Nunez, 1995).

\subsubsection{Results Using $<\mathbf{p}>_{\psi * \psi}$}

$<\mathbf{p}>_{\psi * \psi}$ was used in classical-physics SMNI fits to EEG data using ASA (Ingber, 2016b; Ingber et al., 2014). Runs using $1 \mathrm{M}$ or $100 \mathrm{~K}$ generated states gave results not much different. Training with ASA used $100 \mathrm{~K}$ generated states over 12 subjects with and without $\mathbf{A}$, followed by 1000 generated states with the simplex local code contained with ASA to check precision. Training and Testing runs on XSEDE.org for this project took an equivalent of several months of CPU on the XSEDE.org UCSD San Diego Supercomputer (SDSC) platform Comet. These calculations used one additional parameter across all EEG regions to weight the contribution to synaptic background $B_{G^{\prime}}^{G}$. A is taken to be proportional to the currents measured by EEG, i.e., firings $M^{G}$. Otherwise, the "zerofit-parameter" SMNI philosophy was enforced, wherein parameters are picked from experimentally determined values or within experimentally determined ranges (Ingber, 1984).

As with previous studies using this data, results sometimes gave Testing cost functions less than the Training cost functions. This reflects on great differences in data, likely from great differences in subjects' contexts, e.g., possibly due to subjects' STM strategies only sometimes including effects calculated here. Further tests of these multiple-scale models with more EEG data are required, and with the qPATHINT coupled algorithm described previously. Note that ASA optimizations in this project always include "finishing off" ASA importance-sampling with a modified Nelder-Mead simplex code (included in the ASA code) to ensure best precision. 


\subsubsection{Assumptions for quantum SMNI}

There are assumptions made for this quantum enhancement of SMNI that can only be determined by future experiments.

In the context of quantum mechanics, the wave-function of the $\mathrm{Ca}^{2+}$ wave-packet was calculated, and it was demonstrated that overlap with multiple collisions, due to their regenerative processes, during the observed long durations of hundreds of $\mathrm{ms}$ of typical $\mathrm{Ca}^{2+}$ waves (Ingber, 2015, 2016b, 2017a,c, 2018; Ingber et al., 2014) support a Zeno or "bang-bang" effect (Burgarth et al. 2018 ; Facchi et al., 2004; Facchi \& Pascazio, 2008; Giacosa \& Pagliara, 2014; Kozlowski et al., 2015; Muller et al., 2016; Patil et al., 2015; Wu et al., 2012; Zhang et al. 2014) which may promote long coherence times.

Explicit calculations of reasonable repeated random shocks to the above wave-function $\psi_{F}(t)$ demonstrated only small effects on the repeated projections of this wave-packet after these shocks, i.e., the survival time was calculated (Facchi \& Pascazio, 2008; Ingber, 2018).

Of course, the Zeno/"bang-bang" effect may exist only in special contexts, since decoherence among particles is known to be very fast, e.g., faster than phase-damping of macroscopic classical particles colliding with quantum particles (Preskill, 2015). Here, the constant collisions of $\mathrm{Ca}^{2+}$ ions as they enter and leave the $\mathrm{Ca}^{2+}$ wave-packet due to the regenerative process that maintains the wave, may perpetuate at least part of the wave, permitting the Zeno/"bang-bang" effect. In any case, qPATHINT as used here provides an opportunity to explore the coherence stability of the wave due to serial shocks of this process.

\subsubsection{Nano-Robotic Applications}

Calculations support the possibility of carrying pharmaceutical products in nanosystems that could affect unbuffered $\mathrm{Ca}^{2+}$ waves in neocortex (Ingber, 2015). A Ca ${ }^{2+}$-wave momentum-sensor could act like a piezoelectric device (Ingber, 2018).

The nano-robot could be switched on/off at a regional/columnar level by sensitivity to local electric/magnetic fields. Highly synchronous firings during STM processes can be affected by these piezoelectric nanosystems which affect background/noise efficacies via control of $\mathrm{Ca}^{2+}$ waves. In turn, this would affect the influence of of $\mathrm{Ca}^{2+}$ waves via the vector potential $\mathbf{A}$, etc.

\subsubsection{Free Will}

In addition to the intrinsic interest of researching STM and multiple scales of neocortical interactions via EEG data, there is interest in researching possible quantum influences on highly synchronous neuronal firings relevant to STM to understand possible connections to consciousness and "Free Will" (FW).

As pointed out in some papers (Ingber, 2016a,b), if neuroscience ever establishes experimental feedback from quantum-level processes of tripartite synaptic interactions with large-scale synchronous neuronal firings, that are now recognized as being highly correlated with STM and states of attention, then FW may yet be established using the quantum no-clone "Free Will Theorem" (FWT) (Conway \& Kochen, 2006, 2009).

Basically, this means that a $\mathrm{Ca}^{2+}$ quantum wave-packet may generate a state proven to have not previously existed; quantum states cannot be cloned. In the context of this basic premise, this state may be influential in a large-scale pattern of synchronous neuronal firings, thereby rendering this pattern as a truly new pattern not having previously existed. The FWT shows that this pattern, considered as a measurement of the $\mathrm{Ca}^{2+}$ quantum wave-packet, is correctly identified as itself being a new decision not solely based on previous decisions, even under reasonably stochastic 
experimental and real-life conditions. These considerations require detailed calculations, which are quite different and independent of other philosophical considerations, e.g., as in

https://plato.stanford.edu/entries/qt-consciousness/ .

Only rather recently has the core SMNI hypothesis since circa 1980 (Ingber, 1981, 1982, 1983), that highly synchronous patterns of neuronal firings in fact process high-level information, been verified experimentally (Asher, 2012; Salazar et al., 2012).

\section{3 qPATHINT For SMNI}

An XSEDE.org grant, "Quantum path-integral qPATHTREE and qPATHINT algorithms", was used to test and enhance applications of qPATHTREE and qPATHINT. The wave-function $\psi$ is propagated for its initial state, numerically growing into a tree of wave-function nodes. At each node, interaction of the of $\mathrm{Ca}^{2+}$ wave-packet, via its momentum $\mathbf{p}$, with highly synchronous EEG, via its collective magnetic vector potential $\mathbf{A}$, is calculated to determine changes due to timedependent phenomena. Such changes occur at microscopic scales, e.g., due to modifications of the regenerative wave-packet as ions leave and contribute to the wave-packet, thereby determining the effect on tripartite contributions to neuron-astrocyte-neuron synaptic activity, affecting both $\mathbf{p}$ and A. Such changes also may occur at macroscopic scales, e.g., changes due to external and internal stimuli affecting synchronous firings and thereby A. At every time slice, quantum effects on synaptic interactions are determined by expected values of the interactions over probabilities $\left(\psi^{*} \psi\right)$ determined by the wave-functions at their nodes.

Due to the form of the quantum Lagrangian/Hamiltonian, a multiplicative Gaussian form (with nonlinear drifts and diffusions) is propagated. This permits a straight-forward use of Gaussian quadratures for numerical integration of the expection of the momenta of the wave-packet, i.e., of $<\mathbf{p}(t)>_{\psi * \psi}$. E.g., see

https://en.wikipedia.org/wiki/Gaussian_quadrature

\subsection{Comparing EEG Testing Data with Training Data}

Using EEG data from (Citi et al., 2010; Goldberger et al., 2000)

http://physionet.nlm.nih.gov/pn4/erpbci

SMNI was fit to highly synchronous waves (P300) during attention tasks, for each of 12 subjects, it was possible to find 10 Training runs and 10 Testing runs (Ingber, 2016b). A region of continuous high amplitude of 2561 lines represents times from 17 to 22 secs after the tasks began.

Spline-Laplacian transformations on the EEG potential $\Phi$ are proportional to the SMNI $M^{G}$ firing variables at each electrode site. The electric potential $\Phi$ is experimentally measured by EEG, not $\mathbf{A}$, but both are due to the same currents $\mathbf{I}$. Therefore, $\mathbf{A}$ is linearly proportional to $\Phi$ with a simple scaling factor included as a parameter in fits to data. Additional parameterization of background synaptic parameters, $B_{G^{\prime}}^{G}$ and $B_{E^{\prime}}^{\ddagger E}$, modify previous work.

The $\mathbf{A}$ model outperformed the no- $\mathbf{A}$ model, where the no-A model simply has used $\mathbf{A}$-nondependent synaptic parameters. Cost functions with an $|\mathbf{A}|$ model were much worse than either the A model or the no- $\mathbf{A}$ model. Runs with different signs on the drift and on the absolute value of the drift also gave much higher cost functions than the $\mathbf{A}$ model. 


\subsection{Investigation into Spline-Laplacian Transformation}

As is common practice, codes for the Spline-Laplacian transformations were applied to all electrodes measured on the scalp. However, the PI thinks that the transformation should be applied to each Region of neocortex separately (e.g., visual, auditory, somatic, abstract, etc.), since each region typically participates in attention differently. This process is tested in this project.

\subsection{Summary}

Previous publications have shown how EEG data, under experimental paradigms measuring attention states with varying conditions ranging from studies of alcoholism to different stimuli presented to subjects, can be used to fit the SMNI description of multiple scales of neocortical activity, e.g., including synaptic variables and parameters, collective columnar firings within large regions of neocortex, etc. Recent papers have included models of $\mathrm{Ca}^{2+}$-waves modifying parameterization of background SMNI synaptic parameters. Data was fit to this model using the PI's ASA code (Ingber, 1993, 2012c). These projects demonstrated that fits with this inclusion were better than fits without.

Using qPATHINT, quantum scales of interaction can now be included. A reasonable measure of the influence of these scales is to fit real EEG data under other published experimental paradigms. This is accomplished using the above calculation of $\psi(t)$ with realistic serial shocks, calculating updates to $\mathbf{p}(t)$ and $\mathbf{A}(t)$ at each time slice $t$ corresponding to the EEG data for both training and testing sets of data. While PATHINT could synchronously also be evolved using the SMNI Lagrangian, it likely is convenient and accurate to use the short-term SMNI Lagrangian as in previous studies to fit EEG and at each epoch.

\section{ASA Algorithm}

For parameters

$$
\alpha_{k}^{i} \in\left[A_{i}, B_{i}\right]
$$

sampling with the random variable $x^{i}$

$$
\begin{gathered}
x^{i} \in[-1,1] \\
\alpha_{k+1}^{i}=\alpha_{k}^{i}+x^{i}\left(B_{i}-A_{i}\right)
\end{gathered}
$$

the default generating function is

$$
g_{T}(x)=\prod_{i=1}^{D} \frac{1}{2 \ln \left(1+1 / T_{i}\right)\left(\left|x^{i}\right|+T_{i}\right)} \equiv \prod_{i=1}^{D} g_{T}^{i}\left(x^{i}\right)
$$

in terms of "temperatures" for parameters (Ingber, 1989)

$$
T_{i}=T_{i 0} \exp \left(-c_{i} k^{1 / D}\right)
$$

The default ASA uses the same type of annealing schedule for the acceptance function $h$ as used for the generating function $g$.

All default functions in ASA can be overridden with user-defined functions (Ingber, 1993, 2012c).

ASA has been applied to studies of COVID-19 (Ingber, 2021c). 
ASA has over 150 OPTIONS to provide robust tuning over many classes of nonlinear stochastic systems. These many OPTIONS help ensure that ASA can be used robustly across many classes of systems.

The "QUENCHing" OPTIONS are among the most important for controlling Adaptive Simulated Annealing. Fuzzy ASA algorithms in particular offer ways of controlling how these QUENCHing OPTIONS may be applied across many classes of problems.

In the context of this proposal, ASA has an ASA_SAVE_BACKUP OPTIONS which period ically save all information (including generated random numbers) sufficient to restart if it is interrupted, e.g., using the ASA_EXIT_ANYTIME OPTIONS to simply remove a file "asa_exit_anytime" which causes ASA to gracefully exit. In this project, ASA removes "asa_exit_anytime" each 47 hours.

\section{Path-Integral Methodology}

\subsection{Generic Applications}

There are many systems that are well defined by (a) Fokker-Planck/Chapman-Kolmogorov partialdifferential equations, (b) Langevin coupled stochastic-differential equations, and (c) Lagrangian or Hamiltonian path-integrals. All three such systems of equations are mathematically equivalent, when care is taken to properly take limits of discretized variables in the well-defined induced Riemannian geometry of the system due to nonlinear and time-dependent diffusions (Ingber, 1982, 1983; Langouche et al. 1982; Schulman, 1981).

\subsubsection{Path-Integral Algorithm}

The path integral of a classical system of $\mathrm{N}$ variables indexed by $i$, at multiple times indexed by $\rho$, is defined in terms of its Lagrangian $L$ :

$$
\begin{gathered}
P\left[q_{t} \mid q_{t_{0}}\right] d q(t)=\int \ldots \int D q \exp \left(-\min \int_{t_{0}}^{t} d t^{\prime} L\right) \delta\left(q\left(t_{0}\right)=q_{0}\right) \delta\left(q(t)=q_{t}\right) \\
D q=\lim _{u \rightarrow \infty} \prod_{\rho=1}^{u+1} g^{1 / 2} \prod_{i}(2 \pi \Delta t)^{-1 / 2} d q_{\rho}^{i} \\
L\left(\dot{q}^{i}, q^{i}, t\right)=\frac{1}{2}\left(\dot{q}^{i}-g^{i}\right) g_{i i^{\prime}}\left(\dot{q}^{i^{\prime}}-g^{i^{\prime}}\right)+R / 6 \\
g_{i i^{\prime}}=\left(g^{i i^{\prime}}\right)^{-1}, g=\operatorname{det}\left(g_{i i^{\prime}}\right)
\end{gathered}
$$

Here the diagonal diffusion terms are $g^{i i}$ and the drift terms are $g^{i}$. If the diffusions terms are not constant, then there are additional terms in the drift, and in a Riemannian-curvature potential $R / 6$ for dimension \& 1 in the midpoint Stratonovich/Feynman discretization (Langouche et al., 1982).

The path-integral approach is particularly useful to precisely define intuitive physical variables from the Lagrangian $L$ in terms of its underlying variables $q^{i}$ :

$$
\text { Momentum : } \Pi^{i}=\frac{\partial L}{\partial\left(\partial q^{i} / \partial t\right)}
$$




$$
\begin{gathered}
\text { Mass }: g_{i i^{\prime}}=\frac{\partial L}{\partial\left(\partial q^{i} / \partial t\right) \partial\left(\partial q^{i^{\prime}} / \partial t\right)} \\
\text { Force }: \frac{\partial L}{\partial q^{i}} \\
\mathrm{~F}=\mathrm{ma}: \delta L=0=\frac{\partial L}{\partial q^{i}}-\frac{\partial}{\partial t} \frac{\partial L}{\partial\left(\partial q^{i} / \partial t\right)}
\end{gathered}
$$

E.g., Canonical Momenta Indicators $\left(\mathrm{CMI}=\Pi^{i}\right)$ were used successfully in neuroscience (Ingber, 1996, 1997, 1998), combat analyses (Bowman \& Ingber, 1997), and financial markets (Ingber, 1996; Ingber \& Mondescu, 2001).

The histogram procedure recognizes that the distribution can be numerically approximated to a high degree of accuracy by sums of rectangles of height $P_{i}$ and width $\Delta q^{i}$ at points $q^{i}$. For convenience only, just consider a one-dimensional system. In the prepoint Ito discretization, the path-integral representation can be written in terms of the kernel $G$, for each of its intermediate integrals, as

$$
\begin{gathered}
P(x ; t+\Delta t)=\int d x^{\prime}\left[g^{1 / 2}(2 \pi \Delta t)^{-1 / 2} \exp (-L \Delta t)\right] P\left(x^{\prime} ; t\right)=\int d x^{\prime} G\left(x, x^{\prime} ; \Delta t\right) P\left(x^{\prime} ; t\right) \\
P(x ; t)=\sum_{i=1}^{N} \pi\left(x-x^{i}\right) P_{i}(t) \\
\pi\left(x-x^{i}\right)=1,\left(x^{i}-\frac{1}{2} \Delta x^{i-1}\right) \leq x \leq\left(x^{i}+\frac{1}{2} \Delta x^{i}\right) ; 0, \text { otherwise }
\end{gathered}
$$

This yields

$$
\begin{gathered}
P_{i}(t+\Delta t)=T_{i j}(\Delta t) P_{j}(t) \\
T_{i j}(\Delta t)=\frac{2}{\Delta x^{i-1}+\Delta x^{i}} \int_{x^{i}-\Delta x^{i-1} / 2}^{x^{i}+\Delta x^{i} / 2} d x \int_{x^{j}-\Delta x^{j-1} / 2}^{x^{j}+\Delta x^{j} / 2} d x^{\prime} G\left(x, x^{\prime} ; \Delta t\right)
\end{gathered}
$$

$T_{i j}$ is a banded matrix representing the Gaussian nature of the short-time probability centered about the (possibly time-dependent) drift.

Explicit dependence of $L$ on time $t$ also has been included without complications. Care must be used in developing the mesh $\Delta q^{i}$, which is strongly dependent on diagonal elements of the diffusion matrix, e.g.,

$$
\Delta q^{i} \approx\left(\Delta t g^{i i}\right)^{1 / 2}
$$

This constrains the dependence of the covariance of each variable to be a (nonlinear) function of that variable to present an approximately rectangular underlying mesh. Since integration is inherently a smoothing process (Ingber, 1990), fitting the data with integrals over the short-time probability distribution, this permits the use of coarser meshes than the corresponding stochastic differential equation(s). For example, the coarser resolution is appropriate, typically required, for a numerical solution of the time-dependent path integral. By considering the contributions to the 
first and second moments, conditions on the time and variable meshes can be derived (Wehner \& Wolfer, 1983a). For non-zero drift, the time slice may be determined by a scan of $\Delta t \leq \bar{L}^{-1}$, where $\bar{L}$ is the uniform/static Lagrangian, respecting ranges giving the most important contributions to the probability distribution $P$.

\subsubsection{Direct Kernel Evaluation}

Several projects have used this algorithm (Ingber \& Nunez, 1995; Ingber et al., 1996; Ingber \& Wilson, 1999; Wehner \& Wolfer, 1983a,b, 1987). Special 2-dimensional codes were developed for specific projects in Statistical Mechanics of Combat (SMC) (Ingber et al., 1991), SMNI (Ingber \& Nunez, 1995), and Statistical Mechanics of Financial Markets (SMFM) (Ingber, 2000a).

The previous 1-dimensional PATHINT code was generalized by the PI to be run under N dimensions, by using 'make $\mathrm{D}=\mathrm{N}^{\text {' }}$ in the GCC Makefile. Then, a quantum generalization was made to the code, changing all variables and functions to complex variables. The generic N-dimensional code was developed for classical and quantum systems, using a small shell script called from a Makefile to set up pre-compile options (Ingber, 2016a, 2017a,b).

\subsubsection{Monte Carlo vs Kernels}

Many path-integral numerical applications use Monte Carlo techniques (O'Callaghan \& Miller, 2014). E.g., this approach includes the PI's Adaptive Simulated Annealing (ASA) code using its ASA_SAMPLE OPTIONS (Ingber, 1993). However, this project is concerned with serial (timesequential) random shocks, not conveniently treated with Monte-Carlo/importance-sampling algorithms.

\subsection{Quantum Path Integral Algorithms}

\subsubsection{Scaling Issues}

The use of qPATHINT has been tested with shocks to $\mathrm{Ca}^{2+}$ waves (Ingber, 2017a), using the same basic code used for "contrived" developed for quantum options on quantum money (Ingber, 2017b), serving to illustrate some computational scaling issues, further described in the Performance and Scaling Section.

\subsubsection{Imaginary Time}

Imaginary-time Wick rotations transform imaginary time (the primary source of imaginary dependencies) into a real-variable time. However, when used with numerical calculations, after multiple foldings of the path integral, usually there is no audit trail back to imaginary time to extract phase information (private communication with several authors of path-integral papers, including Larry Schulman on 18 Nov 2015) (Schulman, 1981).

\section{SMNI With qPATHINT}

The above considerations define a clear process of application of fitting EEG using SMNI, with qPATHINT numerically calculating the path-integral at each time between EEG epochs. At the beginning of each EEG epoch, time is reset $(t=0)$ since the wave-function is considered have been decohered ("collapsed") by a prior EEG measurement; until the end of that EEG epoch there are multiple calls to SMNI functions to calculate the evolution of the conditional short-time 
probability distribution, and each call also calls qPATHINT for numerical calculation of the pathintegral, instead of as in previous work using the PI's derived long-time path-integral that has an analytic time-dependent closed-form solution.

\section{$6 \quad$ Performance and Scaling}

Code is used from XSEDE previous XSEDE grant "Electroencephalographic field influence on calcium momentum waves", for SMNI EEG fits. Code is used from XSEDE previous XSEDE grant "Quantum path-integral qPATHTREE and qPATHINT algorithm", for qPATHINT runs.

\subsection{Scaling Estimates}

Estimates below were made on Expanse using 'gcc -O3'. In this context, all debugging flags are not used (e.g., '- g $^{6}$ ) unless specifically noted otherwise, as recommended in

https://www.sdsc.edu/support/user_guides/expanse

\subsubsection{SMNI}

100 ASA-iterations taking 7.12676s yields $0.0712676 \mathrm{sec} / \mathrm{ASA}$-iteration over 2561 EEG epochs. Note that with ' $-\mathrm{g}^{6}$ the total time is $29.9934 \mathrm{~s}$.

The number 2561 of EEG epochs, represents a region of continuous high amplitude of 2561 lines representing times from 17 to 22 secs after the tasks began. This sets time between epochs to be about 0.002 sec.

\subsection{2 $\quad$ qPATHINT}

The current code uses a variable mesh covering 1121 points along the diagonal, with a maximum off-diagonal spread of 27; corners require considerable CPU time to take care of boundaries, etc. Oscillatory wave functions require a large off-diagonal spread (Ingber, 2017a).

If $d t=0.0002$, this requires 10 foldings of the distribution. This takes the code 0.002 s to run, giving $0.0002 /$ qIteraction. Note that with ' $-\mathrm{g}^{6}$ the code takes $0.004 \mathrm{~s}$ to run.

\subsubsection{Projected SUs for This Project}

nSubjects x 2 (switch Train/Test) yields a 24-array set of 1-node jobs.

ASA-iterations x (SMNI_time/ASA-iteration + nEpochs x qIterations x qPATHINT_time/qIteration)

yields $100,000 \times(0.07+2500 \times 10 \times 0.0002)=507,000 \mathrm{sec}=140 \mathrm{hr} / \mathrm{run}=6$ day $/ \mathrm{run}$

where time for the Gaussian quadratures calculations is not appreciable:

https://en.wikipedia.org/wiki/Gaussian_quadrature

Note the maximum duration of a normal job is 2 days. ASA has built in a simple way of ending jobs with printout required to restart, including sets of random numbers generated, so this is quite feasible. 


\subsubsection{XSEDE Ticket with Mahidhar Tatineni}

In tickets.xsede.org \#148054, Mahidhar Tatineni replied:

"Thanks, Lester. That makes it clear. I think in this case using the "shared" partition with array jobs will be perfect and you can restart every 48 hours (make sure you say this in the proposal so that reviewers are aware you can restart).

So you need a total of $24 \times 140=3360$ SUs for each set which is completely reasonable. If you do 100 such sets you will need $350 \mathrm{~K}$ SUs which is completely fine from a request point of view (as long as the runs are justified for the science being done and there is a clear computational plan associated with it).

Mahidhar"

\subsection{Summary of ECSS outcome from 22 July 2020}

Below are quotes from email correspondence concerning my Extended Collaborative Support Services (ECSS) grant to establish whether I have been using XSEDE resources optimally.

"Gujral, Madhusudan to Lester

Dear Lester,

I conveyed your your thanks to Mahi. He is great guy always ready to help even at odd hours. He just communicated to me that node sharing will be available on Expanse as well. You should explain on Scaling and parallelization efficiency section that your application is not multi-threaded and you use single core on comet to run your jobs. This gives efficiency of 1 , which is maximum value achievable. However, you run array of jobs in one submission and each job uses a single core. This is most efficient use of resources because node sharing is allowed on Comet. It won't hurt to write that you have consulted XSEDE staff on this matter.

There might be another unpleasant reason why your proposal gets criticism. People are given 3-weeks to review proposal and many a times people do not get to it till the last day. In such cases, it get cursorily reviewed without attention to details and there is column in the section "is parallel efficiency of the code provided?" which has to be filled. There are situations when code runs on a single core and check column is redundant.

I shall bring up this matter with my manager, Bob Sinkovits, at SDSC when he returns from vacation next week. 
Best, -Madhu"

\section{Conclusion}

A kernel numerical path-integral methodology is presented, to be used with SMNI to fit EEG as a test of the influence of quantum evolution of astrocyte-(re-)generated wave-packets of $\mathrm{Ca}^{2+}$ ions, that suffer shocks due collisions and regeneration of free ions. SMNI is generalized by including quantum variables using qPATHINT. It should be noted that qPATHINT requires large kernel bands for oscillatory states.

The SMNI model has demonstrated it is faithful to experimental data, e.g., STM and EEG recordings under STM experimental paradigms. qPATHINT now permits a new inclusion of quantum scales in the multiple-scale SMNI model, by evolving $\mathrm{Ca}^{2+}$ wave-packets with momentum $\mathbf{p}$, with serial shocks, interacting with the magnetic vector potential $\mathbf{A}$ derived from EEG data, via a $(\mathbf{p}+q \mathbf{A})$ interaction, calculated at each node at each time slice $t$, marching forward in time lock-step with experimental EEG data.

Published pilot studies give a rationale for further developing this particular quantum pathintegral algorithm based on folding kernels, as this can be used to study serial random shocks that occur in many real systems. Furthermore, this quantum version can be used for many quantum systems, which are becoming increasingly important as experimental data is increasing at a rapid pace for many quantum systems.

\section{Acknowledgments}

The PI thanks the Extreme Science and Engineering Discovery Environment (XSEDE.org), for three supercomputer grants since February 2013, "Electroencephalographic field influence on calcium momentum waves", one under PHY130022 and two under TG-MCB140110. A grant under TGMCB140110, "Quantum path-integral qPATHTREE and qPATHINT algorithms", ran through December 2017.

Note that in accordance with common practice, standalone website URLs are not necessarily referenced at the end, but often appear within the body of text. E.g., see

https://libanswers.snhu.edu/faq/8627

https://www.scribbr.com/apa-examples/cite-a-website/

https://libguides.css.edu/APA7thEd/Webpages 


\section{References}

Alakus, T., Gonen, M., Turkoglu, I. (2020). Database for an emotion recognition system based on EEG signals andvarious computer games - GAMEEMO. Biomedical Signal Processing and Control 60(101951), 1-12. https://doi.org/10.1016/j.bspc.2020.101951.

Asher, J. (2012). Brain's code for visual working memory deciphered in monkeys NIH-funded study. Technical Report NIH Press Release, NIH, Bethesda, MD. http://www.nimh.nih.gov/news/ science-news/2012/in-sync-brain-waves-hold-memory-of-objects-just-seen.shtml.

Benedetti, M., Lloyd, E., Sack, S., Fiorentini, M. (2019). Parameterized quantum circuits as machine learning models. Quantum Science and Technology 4(4). https://doi.org/10.1088/ 2058-9565/ab5944.

Bowman, M., Ingber, L. (1997). Canonical momenta of nonlinear combat. In Proceedings of the 1997 Simulation Multi-Conference, 6-10 April 1997, Atlanta, GA, Society for Computer Simulation, San Diego, CA. 1-6. https://www.ingber.com/combat97_cmi.pdf.

Briggs, F., Mangun, G., Usrey, W. (2013). Attention enhances synaptic efficacy and the signal-tonoise ratio in neural circuits. Nature 000,1-5. https://dx.doi.org/10.1038/nature12276.

Burgarth, D., Facchi, P., Nakazato, H., Pascazio, S., Yuasa, K. (2018). Quantum zeno dynamics from general quantum operations. Technical Report arXiv:1809.09570v1 [quant-ph], Aberystwyth U., Aberystwyth, UK.

Citi, L., Poli, R., Cinel, C. (2010). Documenting, modelling and exploiting P300 amplitude changes due to variable target delays in Donchin's speller. Journal of Neural Engineering 7(056006), 1-21. https://dx.doi.org/10.1088/1741-2560/7/5/056006.

Conway, J., Kochen, S. (2006). The free will theorem. Technical Report arXiv:quant-ph/0604079 [quant-ph], Princeton U, Princeton, NJ.

Conway, J., Kochen, S. (2009). The strong free will theorem. Notices of the American Mathematical Society 56(2), 226-232.

Ericsson, K., Chase, W. (1982). Exceptional memory. American Scientist 70, 607-615.

Facchi, P., Lidar, D., Pascazio, S. (2004). Unification of dynamical decoupling and the quantum zeno effect. Physical Review A 69(032314), 1-6.

Facchi, P., Pascazio, S. (2008). Quantum zeno dynamics: mathematical and physical aspects. Journal of Physics A 41(493001), 1-45.

Giacosa, G., Pagliara, G. (2014). Quantum zeno effect by general measurements. Physical Review A 052107, 1-5.

Goldberger, A., Amaral, L., Glass, L., Hausdorff, J., Ivanov, P., Mark, R., Mietus, J., Moody, G., Peng, C.K., Stanley, H. (2000). PhysioBank, PhysioToolkit, and PhysioNet: components of a new research resource for complex physiologic signals. Circulation 101(23), e215-e220. http://circ.ahajournals.org/cgi/content/full/101/23/e215. 
Hick, W. (1952). On the rate of gains of information. Quarterly Journal Experimental Psychology $34(4), 1-33$.

Ingber, L. (1981). Towards a unified brain theory. Journal Social Biological Structures 4, 211-224. https://www.ingber.com/smni81_unified.pdf.

Ingber, L. (1982). Statistical mechanics of neocortical interactions. i. basic formulation. Physica $D$ 5, 83-107. https://www.ingber.com/smni82_basic.pdf.

Ingber, L. (1983). Statistical mechanics of neocortical interactions. dynamics of synaptic modification. Physical Review A 28, 395-416. https://www.ingber.com/smni83_dynamics.pdf.

Ingber, L. (1984). Statistical mechanics of neocortical interactions. derivation of short-term-memory capacity. Physical Review A 29, 3346-3358. https://www.ingber.com/smni84_stm.pdf.

Ingber, L. (1985a). Statistical mechanics of neocortical interactions: Stability and duration of the 7+-2 rule of short-term-memory capacity. Physical Review A 31, 1183-1186. https://www. ingber.com/smni85_stm.pdf.

Ingber, L. (1985b). Statistical mechanics of neocortical interactions. EEG dispersion relations. IEEE Transactions in Biomedical Engineering 32, 91-94. https://www. ingber.com/smni85_eeg.pdf.

Ingber, L. (1989). Very fast simulated re-annealing. Mathematical Computer Modelling 12(8), 967-973. https://www.ingber.com/asa89_vfsr.pdf.

Ingber, L. (1990). Statistical mechanical aids to calculating term structure models. Physical Review A 42(12), 7057-7064. https://www.ingber.com/markets90_interest.pdf.

Ingber, L. (1993). Adaptive simulated annealing (ASA). Technical Report Global optimization C-code, Caltech Alumni Association, Pasadena, CA. https://www.ingber.com/\#ASA-CODE.

Ingber, L. (1994). Statistical mechanics of neocortical interactions: Path-integral evolution of shortterm memory. Physical Review E 49(5B), 4652-4664. https://www.ingber.com/smni94_stm. pdf.

Ingber, L. (1995). Statistical mechanics of neocortical interactions: Constraints on 40 hz models of short-term memory. Physical Review E 52(4), 4561-4563. https://www.ingber.com/smni95_ stm40hz.pdf.

Ingber, L. (1996). Canonical momenta indicators of financial markets and neocortical EEG. In Amari, S.I., Xu, L., King, I., Leung, K.S. (Eds.), Progress in Neural Information Processing, Springer, New York. 777-784. Invited paper to the 1996 International Conference on Neural Information Processing (ICONIP'96), Hong Kong, 24-27 September 1996. ISBN 981-3083-05-0. https://www.ingber.com/markets96_momenta.pdf.

Ingber, L. (1997). Statistical mechanics of neocortical interactions: Applications of canonical momenta indicators to electroencephalography. Physical Review E 55(4), 4578-4593. https: //www.ingber.com/smni97_cmi.pdf.

Ingber, L. (1998). Statistical mechanics of neocortical interactions: Training and testing canonical momenta indicators of EEG. Mathematical Computer Modelling 27(3), 33-64. https://www. ingber.com/smni98_cmi_test.pdf. 
Ingber, L. (1999). Statistical mechanics of neocortical interactions: Reaction time correlates of the g factor. Psycholoquy 10(068). Invited commentary on The g Factor: The Science of Mental Ability by Arthur Jensen. https://www.ingber.com/smni99_g_factor.pdf.

Ingber, L. (2000a). High-resolution path-integral development of financial options. Physica A 283(3-4), 529-558. https://www.ingber.com/markets00_highres.pdf.

Ingber, L. (2000b). Statistical mechanics of neocortical interactions: EEG eigenfunctions of shortterm memory. Behavioral and Brain Sciences 23(3), 403-405. Invited commentary on Toward a Quantitative Description of Large-Scale Neocortical Dynamic Function and EEG, by P.L. Nunez. https://www.ingber.com/smni00_eeg_stm.pdf.

Ingber, L. (2011). Computational algorithms derived from multiple scales of neocortical processing. In Pereira, Jr., A., Massad, E., Bobbitt, N. (Eds.), Pointing at Boundaries: Integrating Computation and Cognition on Biological Grounds, Springer, New York. 1-13. Invited Paper. https://www.ingber.com/smni11_cog_comp.pdf.

Ingber, L. (2012a). Columnar EEG magnetic influences on molecular development of short-term memory. In Kalivas, G., Petralia, S. (Eds.), Short-Term Memory: New Research, Nova, Hauppauge, NY. 37-72. Invited Paper. https://www.ingber.com/smni11_stm_scales.pdf.

Ingber, L. (2012b). Influence of macrocolumnar EEG on ca waves. Current Progress Journal 1(1), 4-8. https://www.ingber.com/smni12_vectpot.pdf.

Ingber, L. (2012c). Adaptive simulated annealing. In Oliveira, Jr., H., Petraglia, A., Ingber, L., Machado, M., Petraglia, M. (Eds.), Stochastic global optimization and its applications with fuzzy adaptive simulated annealing, Springer, New York. 33-61. Invited Paper. https://www.ingber. com/asa11_options.pdf.

Ingber, L. (2015). Calculating consciousness correlates at multiple scales of neocortical interactions. In Costa, A., Villalba, E. (Eds.), Horizons in Neuroscience Research, Nova, Hauppauge, NY. 153-186. ISBN 978-1-63482-632-7. Invited paper. https://www.ingber.com/smni15_calc_ conscious.pdf.

Ingber, L. (2016a). Path-integral quantum PATHTREE and PATHINT algorithms. International Journal of Innovative Research in Information Security 3(5), 1-15. https://www.ingber.com/ path16_quantum_path.pdf.

Ingber, L. (2016b). Statistical mechanics of neocortical interactions: Large-scale EEG influences on molecular processes. Journal of Theoretical Biology 395, 144-152. https://www.ingber.com/ smni16_large-scale_molecular.pdf.

Ingber, L. (2017a). Evolution of regenerative ca-ion wave-packet in neuronal-firing fields: Quantum path-integral with serial shocks. International Journal of Innovative Research in Information Security 4(2), 14-22. https://www.ingber.com/path17_quantum_pathint_shocks.pdf.

Ingber, L. (2017b). Options on quantum money: Quantum path-integral with serial shocks. International Journal of Innovative Research in Information Security 4(2), 7-13. https: //www.ingber.com/path17_quantum_options_shocks.pdf.

Ingber, L. (2017c). Quantum path-integral qPATHINT algorithm. The Open Cybernetics Systemics Journal 11, 119-133. https://www.ingber.com/path17_qpathint.pdf. 
Ingber, L. (2018). Quantum calcium-ion interactions with EEG. Sci 1(7), 1-21. https://www. ingber.com/smni18_quantumCaEEG.pdf.

Ingber, L. (2021a). Revisiting our quantum world. In Hooke, A. (Ed.), Technological Breakthroughs and Future Business Opportunities in Education, Health, and Outer Space, IGI Global, Hershey, PA. (to be published). https://www.ingber.com/quantum20_techcast.pdf.

Ingber, L. (2021b). Quantum calcium-ion affective influences measured by EEG. International Journal Physics and Space Science , (to be published)https://www.ingber.com/quantum20_ affective.pdf.

Ingber, L. (2021c). Forecasting COVID-19 with importance-sampling and path-integrals. WSEAS Transactions on Biology and Biomedicine, (to be published)https://www.ingber.com/asa20_ covid.pdf.

Ingber, L., Chen, C., Mondescu, R., Muzzall, D., Renedo, M. (2001). Probability tree algorithm for general diffusion processes. Physical Review E 64(5), 056702-056707. https://www . ingber. com/path01_pathtree.pdf.

Ingber, L., Fujio, H., Wehner, M. (1991). Mathematical comparison of combat computer models to exercise data. Mathematical Computer Modelling 15(1), 65-90. https://www.ingber.com/ combat91_data.pdf.

Ingber, L., Mondescu, R. (2001). Optimization of trading physics models of markets. IEEE Transactions Neural Networks 12(4), 776-790. Invited paper for special issue on Neural Networks in Financial Engineering. https://www.ingber.com/markets01_optim_trading.pdf.

Ingber, L., Nunez, P. (1995). Statistical mechanics of neocortical interactions: High resolution path-integral calculation of short-term memory. Physical Review E 51(5), 5074-5083. https: //www.ingber.com/smni95_stm.pdf.

Ingber, L., Nunez, P. (2010). Neocortical dynamics at multiple scales: EEG standing waves, statistical mechanics, and physical analogs. Mathematical Biosciences 229, 160-173. https: //www.ingber.com/smni10_multiple_scales.pdf.

Ingber, L., Pappalepore, M., Stesiak, R. (2014). Electroencephalographic field influence on calcium momentum waves. Journal of Theoretical Biology 343, 138-153. https://www.ingber.com/ smni14_eeg_ca.pdf.

Ingber, L., Srinivasan, R., Nunez, P. (1996). Path-integral evolution of chaos embedded in noise: Duffing neocortical analog. Mathematical Computer Modelling 23(3), 43-53. https://www. ingber.com/path96_duffing.pdf.

Ingber, L., Wilson, J. (1999). Volatility of volatility of financial markets. Mathematical Computer Modelling 29(5), 39-57. https://www.ingber.com/markets99_vol.pdf.

Ingber, L., Wilson, J. (2000). Statistical mechanics of financial markets: Exponential modifications to black-scholes. Mathematical Computer Modelling 31(8/9), 167-192. https://www.ingber. com/markets00_exp.pdf.

Jensen, A. (1987). Individual differences in the hick paradigm. In Vernon, P. (Ed.), Speed of Information-Processing and Intelligence, Ablex, Norwood, NJ. 101-175. 
Kozlowski, W., Caballero-Benitez, S., Mekhov, I. (2015). Non-hermitian dynamics in the quantum zeno limit. Technical Report arXiv:1510.04857 [quant-ph], U Oxford, Oxford, UK.

Langouche, F., Roekaerts, D., Tirapegui, E. (1982). Functional Integration and Semiclassical Expansions. Reidel, Dordrecht, The Netherlands.

Mountcastle, V., Andersen, R., Motter, B. (1981). The influence of attentive fixation upon the excitability of the light-sensitive neurons of the posterior parietal cortex. Journal of Neuroscience $1,1218-1235$.

Muller, M., Gherardini, S., Caruso, F. (2016). Quantum zeno dynamics through stochastic protocols. Technical Report arXiv:1607.08871v1 [quant-ph], U Florence, Florence, Italy.

Murakami, S., Okada, Y. (2006). Contributions of principal neocortical neurons to magnetoencephalography and electroencephalography signals. Journal of Physiology 575(3), 925-936.

Nunez, P., Srinivasan, R. (2006). Electric Fields of the Brain: The Neurophysics of EEG, 2nd Ed. Oxford University Press, London.

Nunez, P., Srinivasan, R., Ingber, L. (2013). Theoretical and experimental electrophysiology in human neocortex: Multiscale correlates of conscious experience. In Pesenson, M. (Ed.), Multiscale Analysis and Nonlinear Dynamics: From genes to the brain, Wiley, New York. 149-178. https: //doi.org/10.1002/9783527671632.ch06.

O'Callaghan, M., Miller, B. (2014). Path integral monte carlo on a lattice: Extended states. Physical Review E 89(042124), 1-19. https://dx.doi.org/10.1103/PhysRevE.89.042124.

Patil, Y., Chakram, S., Vengalattore, M. (2015). Measurement-induced localization of an ultracold lattice gas. Physical Review Letters 115(140402), 1-5. http://link.aps.org/doi/10.1103/ PhysRevLett.115.140402.

Preskill, J. (2015). Quantum mechanics. Technical Report Lecture Notes, Caltech, Pasadena, CA. http://www.theory.caltech.edu/people/preskill/ph219/.

Ross, W. (2012). Understanding calcium waves and sparks in central neurons. Nature Reviews Neuroscience 13, 157-168.

Salazar, R., Dotson, N., Bressler, S., Gray, C. (2012). Content-specific fronto-parietal synchronization during visual working memory. Science 338(6110), 1097-1100. https://dx.doi.org/10. $1126 /$ science.1224000.

Schulman, L. (1981). Techniques and Applications of Path Integration. J. Wiley \& Sons, New York.

Schulten, K. (1999). Quantum mechanics. Technical Report Lecture Notes, U. Illinois, Urbana, IL. http://www.ks.uiuc.edu/Services/Class/PHYS480/.

Wehner, M., Wolfer, W. (1983a). Numerical evaluation of path-integral solutions to fokker-planck equations. I. Physical Review A 27, 2663-2670.

Wehner, M., Wolfer, W. (1983b). Numerical evaluation of path-integral solutions to fokker-planck equations. II. restricted stochastic processes. Physical Review A 28, 3003-3011.

Wehner, M., Wolfer, W. (1987). Numerical evaluation of path integral solutions to fokker-planck equations. III. time and functionally dependent coefficients. Physical Review A 35, 1795-1801. 
Wu, S., Wang, L., Yi, X. (2012). Time-dependent decoherence-free subspace. Journal of Physics A 405305, 1-11.

Zhang, G., Simon, H. (1985). STM capacity for chinese words and idioms: Chunking and acoustical loop hypotheses. Memory $\&$ Cognition 13, 193-201.

Zhang, P., Ai, Q., Li, Y., Xu, D., Sun, C. (2014). Dynamics of quantum zeno and anti-zeno effects in an open system. Science China Physics, Mechanics \& Astronomy 57(2), 194-207. https://dx.doi.org/10.1007/s11433-013-5377-x. 